\title{
Tratamento do sorriso gengival com toxina botulínica tipo A: relato de caso
}

Treatment of gummy smile using botulinum toxin type A: case report

Alessandra Kuhn-Dall'Magro* Suélen Cristina Calza**

Jonathan Lauxen ${ }^{* * *}$

Renato dos Santos ${ }^{* * * *}$

Túlio del Conte Valcanaia*****

Eduardo Dall'Magro ${ }^{* * * * * *}$

\section{Resumo}

Objetivo: o sorriso é a mais bela das expressões faciais e está diretamente relacionado com bem-estar, felicidade, prazer entre outros. Fisiologicamente é o resultado da exposição dos dentes e da gengiva durante a contração de um grupo muscular. Muitas pessoas ao sorrir mostram uma quantidade excessiva de gengiva, o que constitui um problema estético. Assim, este artifo apresenta uma revisão de literatura a respeito da etiologia e do tratamento do sorriso gengival, bem como, relata por meio de caso clínico a indicação da toxina botulínica como modalidade de tratamento para esse. Relato de caso: paciente do sexo feminino, 22 anos, com sorriso gengival causado por hipermobilidade dos músculos elevadores do lábio superior, exposição gengival bilateral de $4 \mathrm{~mm}$. Foi tratada com $10 \mathrm{U}$ bilaterais de toxina botulínica tipo A que resultou em um sorriso harmônico e esteticamente mais agradável. Considerações finais: $o$ sorriso gengival é considerado uma desordem estética por muitos pacientes e pode ser tratado por meio da utilização da toxina botulínica.

Palavras-chave: Sorriso gengival. Toxina botulínica tipo A. Estética.

\section{Introdução}

O sorriso de um paciente pode exprimir sensação de alegria, êxito, sensualidade, afeto, cortesia. Um sorriso é mais que uma forma de comunicação, ele é meio de socialização e atração. É indiscutível que a profissão que mais convive com e manipula sorrisos é a Odontologia. Entretanto, frequentemente, cirurgiões-dentistas (CDs), especialistas ou não, negligenciam em seus planejamentos considerações fundamentais a respeito dos padrões de sorriso $^{1}$. Do ponto de vista anatômico e fisiológico, o sorriso é o resultado da exposição dos dentes e da gengiva durante a contração dos grupos musculares dos terços médio e inferior da face ${ }^{2}$. Um sorriso harmônico é determinado não só pela forma, posição e cor dos dentes, mas também pelo tecido gengival ${ }^{3}$.

O primeiro passo para estabelecer um diagnóstico correto de um sorriso harmônico é classificar adequadamente o nível gengival, respeitando-se variáveis, como gênero, idade e saúde periodontal. Uma vez determinada a anormalidade do nível do sorriso, o estabelecimento da sua etiologia é impres-

Especialista em Cirurgia e Traumatologia Bucomaxilofacial (CTBMF), Santa Casa, Porto Alegre; Mestre em Ciências Médicas - Faculdade de Medicina-UFRGS; Professora dos Cursos de Pós Graduação em CTBMF do Ceom (Centro de Estudos Odontológicos Meridional) - Passo Fundo e do IOA (Instituto Odontológico das Américas) -Balneário Camboriú -SC; Membro do Corpo Clínico do Hospital São Vicente de Paulo de Passo Fundo (HSVP) e do IOT (Instituto de Ortopedia e Traumatologia), Passo Fundo - RS, Brasil.

Cirurgiã-Dentista graduada pela Faculdade de Odontologia da Uniplac, Lages - SC, Brasil; Aluna do Curso de Especialização em Dentística do CEOM, Passo Fundo - RS, Brasil.

*** Cirurgião-Dentista graduado pela Faculdade de Odontologia da Universidade de Passo Fundo (UPF), Passo Fundo - RS, Brasil.

***** Especialista em CTBMF-CEOM - HSVP, Passo Fundo - RS, Brasil.

***** Especialista e Mestre em CTBMF, Universidade Federal de Pelotas; Doutor em CTBMF - Pontifícia Universidade Católica do Rio Grande do Sul (PUCRS); Professor Titular da Universidade do Vale do Itajaí (Univali); Professor de CTBMF da Faculdade Avantis; Coordenador do Curso de Especialização em Implantodontia da Faculdade Avantis.

****** Especialista em Prótese Dentária - USP, Mestre em Laser em Odontologia - USP, Mestre e Doutor em Materiais Dentários - Unicamp. Professor Titular III da Faculdade de Odontologia da UPF, Passo Fundo - RS, Brasil. 
cindível. Geralmente, esta ocorre de maneira multifatorial relacionando-se, principalmente, ao crescimento vertical excessivo da maxila, comprimento reduzido do lábio superior, contração excessiva do lábio superior e desproporção comprimento/largura da coroa clínica dos dentes anteriores. Para alguns autores a extrusão dos dentes superiores, associada à mordida profunda também é um fator que pode estar relacionado ao problema ${ }^{1,4-6}$.

Uma vez estabelecido o diagnóstico do sorriso gengival, uma modalidade de tratamento minimamente invasivo, que pode servir como adjuvante ou substituto para o procedimento cirúrgico é o uso de toxina botulínica (BTX). Esta toxina atua aderindo à proteína sinaptosômica (SNAP-25) e inibindo a liberação de acetilcolina, impedindo, desse modo, a contração muscular. Entre os sete tipos de neurotoxina botulínicas sorologicamente disponíveis, a BTX-A é mais frequentemente utilizada ${ }^{1,7-9}$. O objetivo deste trabalho é realizar uma revisão de literatura a respeito da etiologia e do tratamento do sorriso gengival, além de relatar, por meio de caso clínico, a indicação da BTX como modalidade de tratamento.

\section{Revisão de literatura}

De acordo com Pascotto e Moreira $(2005)^{10}$, ao sorrir o lábio superior move-se apicalmente, expondo os dentes anteriores e margens gengivais. Nessa situação, normalmente, 1 a $2 \mathrm{~mm}$ de gengiva ficam aparentes. Quando mais de $2 \mathrm{~mm}$ de gengiva é exposta, durante o sorriso, caracteriza-se a situação denominada "sorriso gengival". Apesar de casos de sorriso gengival aparecerem com certa frequência nos consultórios odontológicos, a literatura dedicada a esse assunto como tema central, abordando seu diagnóstico e tratamento, é escassa ${ }^{11}$.

A percepção estética de um sorriso gengival foi comparada por diferentes categorias de indivíduos no estudo feito por Suzuki et al. $(2009)^{12}$. Foram utilizadas para as avaliações fotografias do sorriso de quatro indivíduos, dois homens e duas mulheres. Cada fotografia original foi manipulada no computador para a criação de cinco imagens, com diferentes graus de exposições gengivais: 0, 1, 3, 5 e $7 \mathrm{~mm}$. Em seguida, as imagens foram submetidas à avaliação de sessenta indivíduos, divididos igualmente em três categorias, ortodontistas, cirurgiões buco-maxilo-faciais e leigos, que atribuíram notas de 0 a 10 (onde zero era considerado o sorriso desagradável e dez o extremamente agradável). Os resultados demonstraram que as exposições gengivais de 0 e 1 $\mathrm{mm}$ receberam as maiores pontuações estéticas, demonstrando que este nível de exposição é atraente para todos os grupos.

Em estudo comparativo para avaliar a influência da quantidade de exposição gengival na estética do sorriso para os sexos masculino e feminino, foram convidados ortodontistas, clínicos gerais e leigos para avaliar fotografias da face durante $o$ sorriso com alterações digitais que produziam cinco diferentes níveis de exposição gengival. Tais alterações variaram de $4 \mathrm{~mm}$ de exposição a $4 \mathrm{~mm}$ de cobertura dos incisivos superiores. Concluíram que a atratividade do sorriso é influenciada pela quantidade de exposição gengival. $\mathrm{O}$ sorriso mais estético para o sexo feminino e masculino foi aquele em que o lábio repousa sobre a margem cervical dos incisivos superiores ${ }^{13}$.

Assim, a posição e a quantidade de dentes e gengiva exposta durante o sorriso e a fala, respeitandose o dimorfismo sexual e os efeitos da idade são critérios fundamentais, dinâmicos e imprescindíveis para o diagnóstico e o planejamento multidisciplinar, sob pena de descaracterizarmos os pacientes em função de sua idade e gênero ${ }^{1,4,14}$. Ao exame clínico/fotográfico dois pontos-chave devem ser considerados quando se busca determinar a visibilidade do periodonto. Primeiramente, o CD deve olhar não para a visibilidade da gengiva marginal (a etapa antes do sorriso), mas também na visibilidade das papilas gengivais. Em segundo lugar, o profissional deve considerar ambos, o sorriso natural e o sorriso forçado ao avaliar a posição da linha de sorriso. Quando é solicitado para um paciente sorrir, ele, normalmente, toma uma atitude cautelosa e revela um sorriso mais ou menos natural. Contudo, fora do consultório, o paciente pode revelar mais periodonto forçando o sorriso ao grau máximo de contração de lábio, fazendo por meio disso o sorriso menos estético. Razão pela qual recomenda-se $o$ uso de filmagem ou a observação das expressões do paciente.

\section{Etiologia e tratamento do sorriso gengival}

Vários fatores etiológicos têm sido propostos. Didaticamente pode-se dividí-los em: dentário, gengival, ósseo e muscular.

Dentário: Quando a exposição excessiva de gengiva ao sorrir for de etiologia dentária, ter-se-á extrusão excessiva dos incisivos superiores, caracterizando overbite, a qual poderá ser tratada apenas com mecânica intrusiva. Casos tratados com mecânica intrusiva apoiada em minimplantes, geralmente são associados à cirurgia periodontal ao final do tratamento ortodôntico ${ }^{1,5,6}$. Além disso, durante a análise dentária precisamos prestar atenção no plano oclusal do paciente e no quanto ele contribui para o sorriso gengival (Figura 1). 


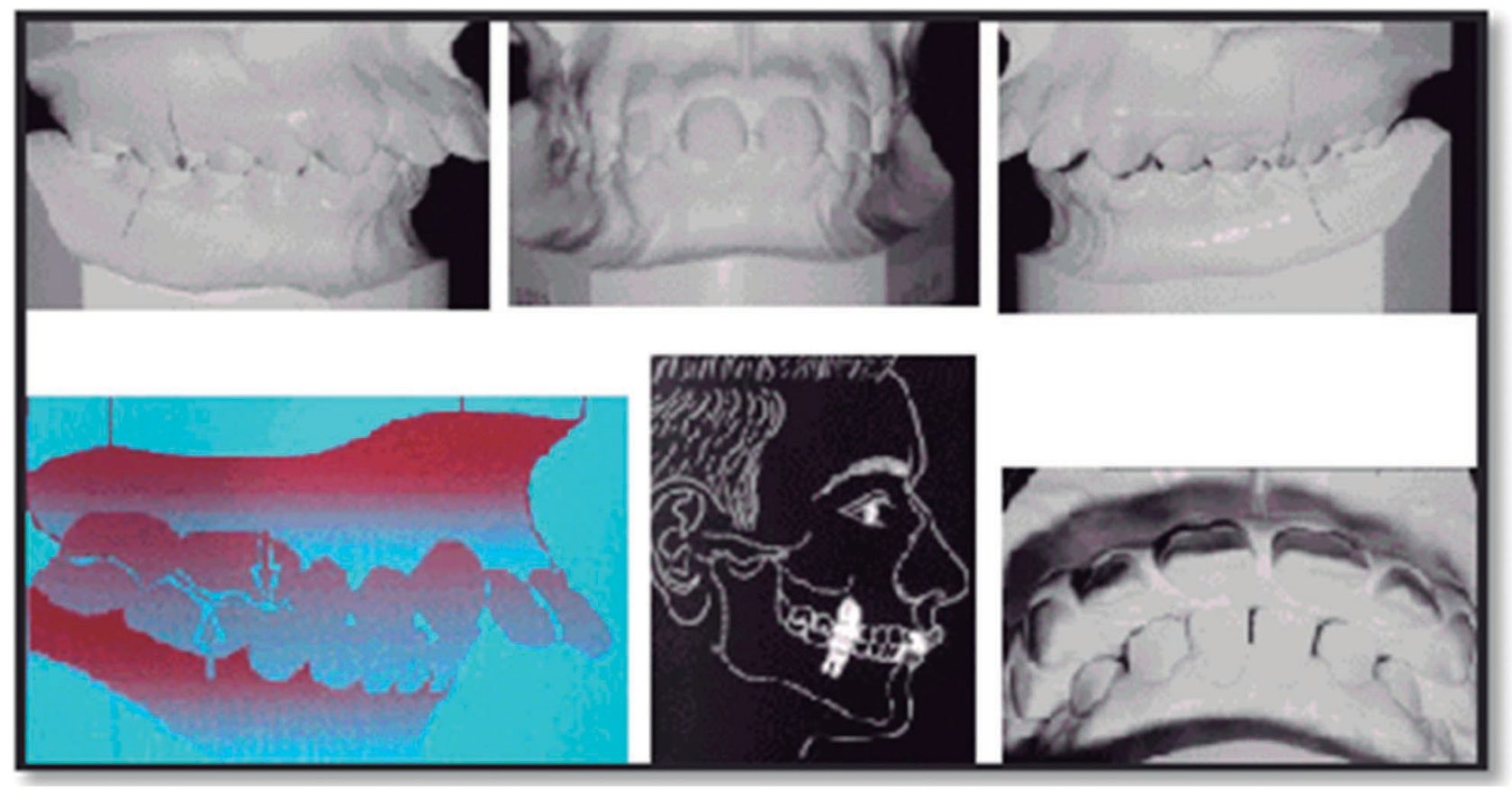

Figura 1 - Avaliação dentária e oclusal do paciente

Gengival: A desproporção altura/largura da coroa clínica é, frequentemente, indicativo de problema associado a excesso gengival mais localizado, como nos casos de erupção passiva, ou mais generalizado, como nos casos de crescimento hiperplásico. O ortodontista, com sua mecânica, tráz poucos im- pactos positivos na solução desse tipo de problema. Apesar disso, durante o tratamento de más oclusões que estejam associadas a esses, deve-se ter atenção redobrada ao planejamento multidisciplinar estabelecendo a hora e o momento certo para o tratamento periodontal ${ }^{1,9,15}$ (Figura 2).
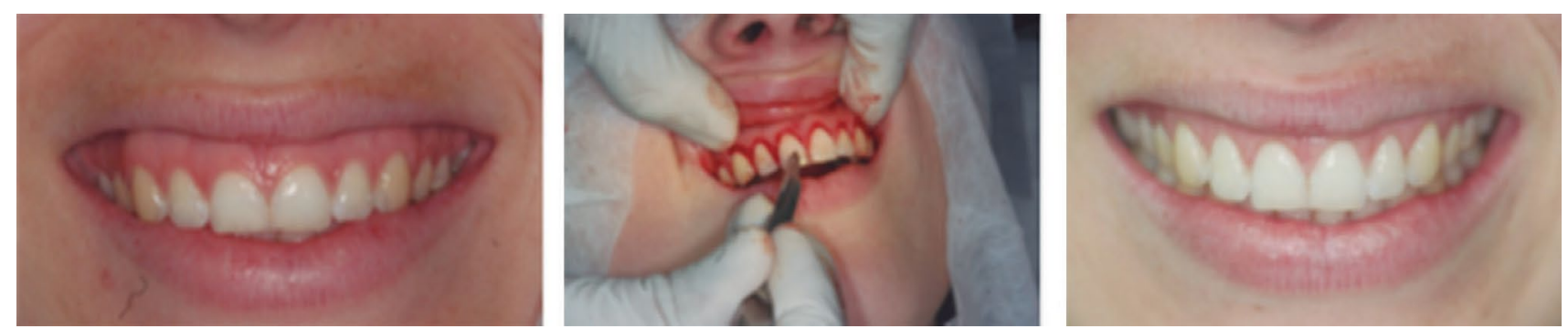

Figura 2 - Sorriso gengival decorrente de hiperplasia gengival (Pré, trans e pós-operatório)

Ósseo: A estrutura óssea é avaliada por meio de cefalometria. A etiologia óssea caracterizada pelo excesso vertical maxilar, manifesta-se principalmente em pacientes com crescimento predominantemente vertical. Clinicamente, observa-se um sorriso gengival anterior e posterior, sem a presença de excesso gengival. Nesse caso, o tratamento do crescimento vertical excessivo implica indubitavelmente cirurgia ortognática ${ }^{1,16}$ (Figura 3 ).

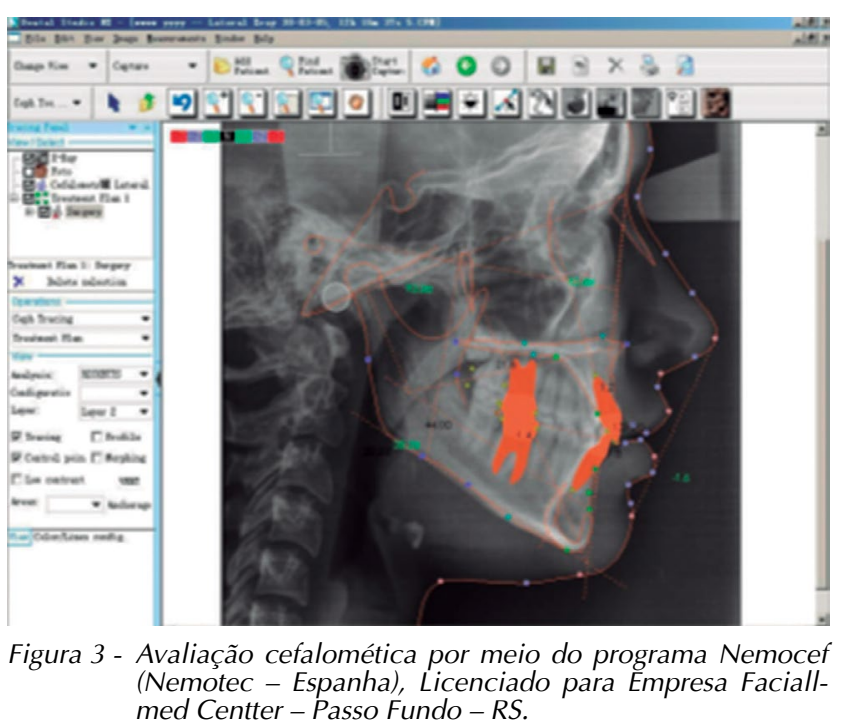


Muscular: Por fim, a análise muscular é conduzida. Observa-se o comprimento e a contração labial ao sorriso espontâneo. Quando todos os fatores anteriores, inclusive o comprimento labial, estão normais, a única causa possível torna-se, então, a hipercontração labial. É justamente no campo muscular, principalmente nos casos de comprimento labial normal - que encontramos a hipercontração dos músculos elevadores do lábio determinando o sorriso gengival de forma que o tratamento é a indicação de uso da toxina botulínica (BTX), uma proposta recente de ferramenta dos $\operatorname{CDs}^{1,2,7,9,17}$ (Figura 4).

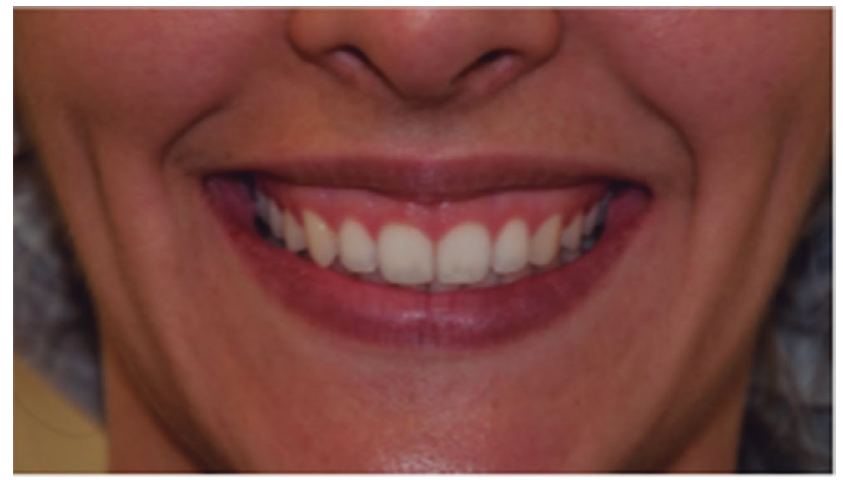

Figura 4 - Sorriso gengival decorrente de hipercontração muscular

\section{Toxina botulínica tipo A (BTX-A)}

Apesar de a BTX-A ser amplamente conhecida por sua utilização cosmética, a sua principal aplicação é voltada ao uso terapêutico. A utilização dessa toxina purificada em procedimentos cosméticos só foi aprovada pela Anvisa, no Brasil, em 2000 e, nos EUA, pela FDA, em 2002. A utilização terapêutica da BTX foi primeiramente estudada por Scott e colaboradores em 1973, em primatas. No final da década de 1970, a toxina foi introduzida como um agente terapêutico para o tratamento do estrabismo. Desde então suas aplicações terapêuticas têm sido ampliadas em diferentes campos ${ }^{18}$. Na Odontologia brasileira a BTX-A foi devidamente regulamentada para uso pela resolução 112/11 do Conselho Federal de Odontologia desde setembro de $2011^{19}$. Por ter conhecimento sobre as estruturas de cabeça e pescoço, o CD pode tratar certas afecções da face e da cavidade oral de forma conservadora e segura com a aplicação da BTX-A, desde que tenha treinamento específico e conhecimento sobre sua utilização e não extrapole sua área de atuação ${ }^{18}$.

A BTX é produzida pela bactéria anaeróbia Clostridium botulinum. Existem sorotipos diferentes de toxina botulínica. A do tipo A (BTX-A) é a mais potente e mais utilizada clinicamente. O produto é um pó estável seco por vácuo, que é diluído com solução salina estéril sem conservantes ${ }^{17}$.

A toxina age impedindo a liberação de acetilcolina a partir das vesículas pré-sinápticas na junção neuromuscular, resultando na inibição da contração muscular. Esse bloqueio é temporário, variando de três a quatro meses, após o que, ocorre o surgimento de novos terminais do axônio, resultando em um retorno da função neuromuscular. Portanto, o tratamento com a BTX-A não pode ser considerado curativo, mas uma abordagem paliativa e sintomática para a gestão de um problema ${ }^{20}$.

A BTX-A tem sido amplamente utilizada na odontologia para o tratamento de hiperfunção muscular como é o caso do sorriso gengival, além de desordens temporomandibulares, assimetrias faciais, hipertrofia massetérica, espasmo hemifacial, dor miofascial, sialorréia e bruxismo ${ }^{21-23}$.

Uma grande vantagem dessa abordagem de tratamento é que é $100 \%$ reversível, assim, se houver qualquer aspecto de mudança de aparência sobre a qual o paciente não esteja contente, ele pode ter certeza de que o efeito é temporário ${ }^{21}$.

Segundo Jankovic (2004) ${ }^{24}$, que investigou a prática clínica da BTX-A, a latência média do tempo da injeção para o início da manifestação clínica é de três a cinco dias e a duração do benefício é, geralmente, de três a quatro meses.

\section{Relato de caso}

Paciente do sexo feminino, 22 anos, procura atendimento em decorrência do excesso de exposição gengival ao sorrir. Após exame diagnóstico completo, envolvendo análise das estruturas dentárias, gengivais, esqueléticas e musculares do sorriso, constatou-se sorriso gengival por hipermobilidade dos músculos elevadores do lábio superior. A paciente apresentava durante o sorriso forçado uma exposição gengival bilateral de $4 \mathrm{~mm}$. Após assinatura do Termo de Consentimento Livre e Esclarecido, a paciente opta por tratamento do seu sorriso gengival por meio da BTX-A (Figuras 5 a 8).

\section{Protocolo de Tratamento:}

1- anamnésico /exame inicial: $4 \mathrm{~mm}$ de exposição gengival bilateral;

2- assinatura do Termo de Consentimento Informado e Esclarecido;

3- fotografias / filmagem do caso;

4- higienização da face;

5- demarcação da face (lapis tinta);

6- anestesia: anestésico tópico - EMLA (lidocaína 25 $\mathrm{mg} / \mathrm{g}$; prilocaina $25 \mathrm{mg} / \mathrm{g}$ AstraZeneca);

7- aplicação do produto (área demarcada); 10 unidades (U) de cada lado (ao lado da narina no sulco naso-labial);

8- recomendações: cabeça elevada por $4 \mathrm{~h}$; não comprimir a região; não realizar atividades físicas por $24 \mathrm{~h}$;

9- agendamento da revisão (Fotografias finais): entre 15 e 30 dias. 


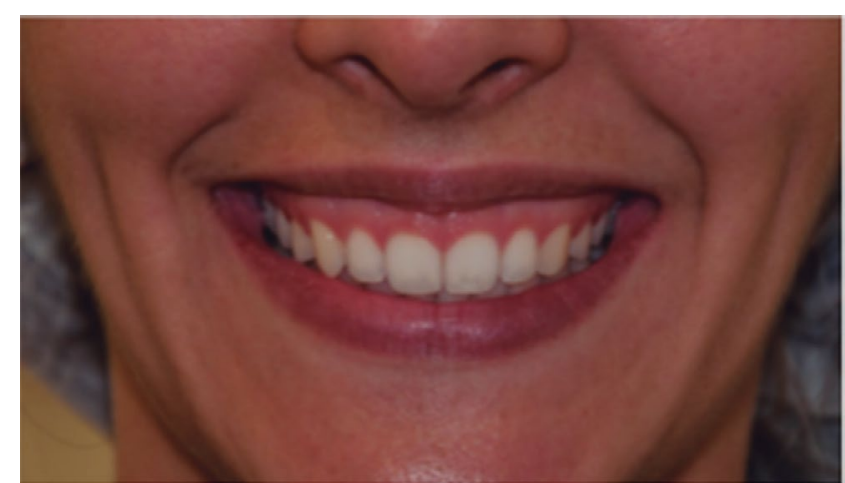

Figura 5 - Sorriso gengival com $4 \mathrm{~mm}$ de exposição bilateral (antes da intervenção)

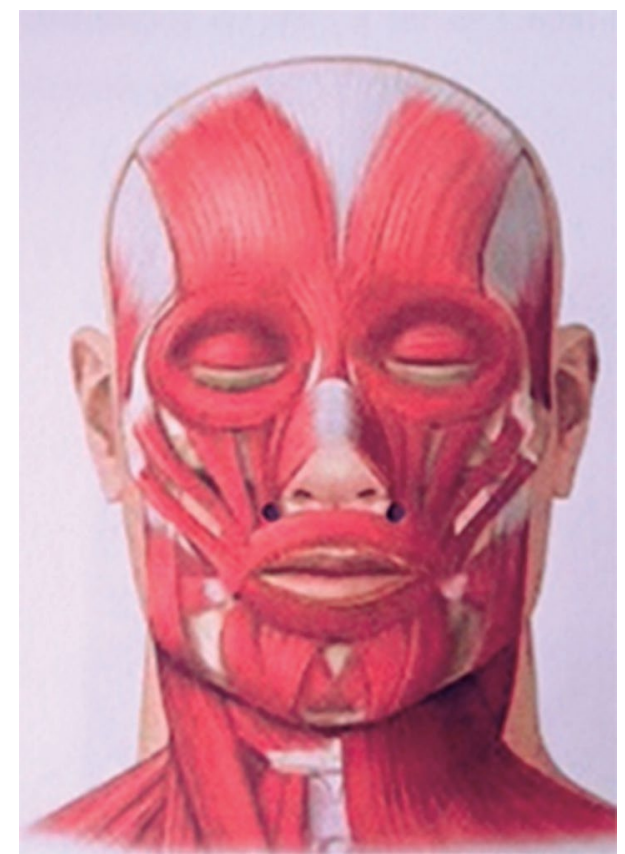

Figura 6 - Pontos de Aplicação da BTX-A (10 U de cada lado)

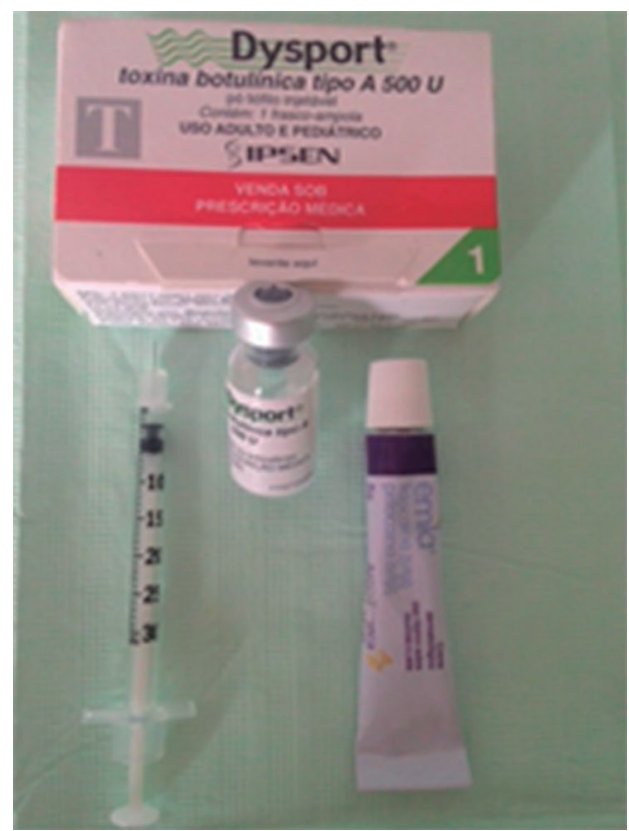

Figura 7 - BTX-A - Produto reconstituído com 1,1 m/ de soro fisiológico; seringa indicando a mensuração de $10 \mathrm{U}$ a ser aplicada; EMLA: anestésico tópico, usualmente aplicado 30 min antes da intervenção .

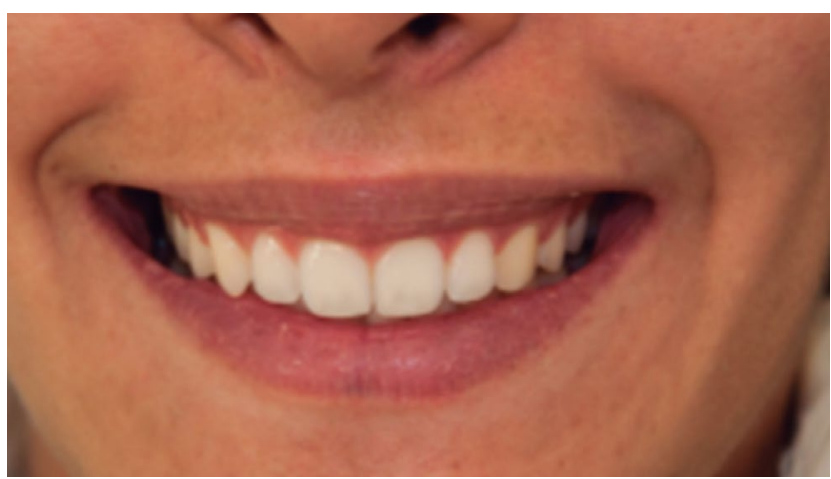

Figura 8 - Paciente reavaliada após 30 dias de aplicação da BTX-A

\section{Discussão}

Uma das queixas principais dos pacientes que procuram a Odontologia estética é o excesso gengival. Uma vez diagnosticada sua etiologia, o CD dispõe dos recursos necessários para o seu tratamento. No caso de sorriso gengival atribuído a uma hiperfunção do músculo elevador do lábio superior, como o relatado neste estudo, a BTX-A pode ser indicada. Na última década muitos trabalhos foram realizados no intuito de se avaliar o comportamento da BTX-A, avaliando dosimetria, efeito e duração dos resultados.

Polo $(2005)^{17}$, com o propósito de determinar se a BTX-A também poderia ser utilizada em pacientes com hiperfunção do músculo elevador do lábio superior para corrigir o sorriso gengival, fez um estudo inicial com cinco indivíduos, nos quais realizou aplicações da BTX-A e obteve como resultado a melhoria temporária de sorrisos gengivais por meio dessa técnica. Segundo ele, estudos futuros seriam necessários para avaliar esse tratamento em amostras maiores.

Em 2008, Polo ${ }^{25}$ pesquisou 30 indivíduos com exposição gengival que receberam injeções de BTX-A. Os pacientes avaliaram os efeitos e também foram avaliados por clínicos especialistas após $2,4,8,12$, 16, 20 e 24 semanas. A redução média do lábio em 2 semanas foi de $5,1 \mathrm{~mm}$ para 30 pacientes. A exposição aumentou gradualmente, a partir de duas semanas pós-injeção, ao longo de 24 semanas, mas, nesse período, a exposição média gengival não havia retornado aos valores basais. Com base em previsões de uma equação polinomial de terceiro grau, a média inicial de 5,2 $\mathrm{mm}$ não seria alcançada até as 30 ou 32 semanas de pós-operatório. Concluiu-se que as injeções de BTX para a correção neuromuscular do sorriso gengival causadas por hiperfunção dos músculos elevadores do lábio superior foi um procedimento eficaz e estatisticamente superior ao da linha de base do sorriso, embora o efeito seja transitório.

No estudo realizado por Hwang et al. (2009) $)^{7}$ foram utilizados 50 hemi-faces de 25 cadáveres adultos (treze do sexo masculino e doze do sexo feminino), nos quais foram investigadas as relações 
topográficas e as direções dos três músculos responsáveis pelo sorriso: levantador do lábio superior, levantador do lábio superior e da asa do nariz e zigomático menor, com o propósito de encontrar um ponto de injeção seguro e reprodutível para a BTX. Utilizaram marcações em determinados pontos de referências a partir dos quais foram traçados círculos com um raio de $1 \mathrm{~cm}$ em cada ponto e ligados entre si formando uma área triangular. Observou-se que os três vetores musculares passavam no centro do triangulo. Assim, o centro desse triangulo foi chamado de "Ponto de Yonsei" e sugerido como um ponto de injeção apropriado para a aplicação da BTX-A. Também foram calculadas as distâncias do "Ponto de Yonsei" à asa do nariz e à linha do lábio, a média horizontal foi de $2,1 \mathrm{~mm}$ em homens e mulheres, já a média vertical foi de $4,2 \mathrm{~mm}$ em homens e $3,3 \mathrm{~mm}$ em mulheres. A eficácia clínica do ponto de injeção foi demonstrada em casos selecionados com ou sem tratamento ortodôntico, concluindo que a BTX-A pode ser uma alternativa de tratamento eficaz para pacientes com exposição gengival excessiva causada pela hiperatividade dos músculos elevadores do lábio.

Segundo Indra et al. $(2011)^{26}$, ao contrário de vários outros procedimentos cirúrgicos, a BTX provou ser uma alternativa minimamente invasiva, eficaz para a correção do sorriso gengival causado pelo músculo elevador do lábio superior. No estudo de um caso de excesso vertical de maxila grave, que foi tratado em conjunto com osteotomia Lefort-I, plastia V-Y para alongamento de lábio e BTX-A, puderam concluir que a BTX-A pode ser um complemento útil para realçar a estética e melhorar a satisfação do paciente em que a cirurgia ortognática, como técnica isolada, pode revelar-se insuficiente.

Os protocolos atuais de aplicação da BTX-A preconizam a aplicação de $10 \mathrm{U}$ bilateralmente na região do sulco naso-labial adjacente à asa do nariz conforme demostrado no presente relato de caso. Doses adicionais de BTX-A poderão ser realizadas entre 15 e 30 dias da primeira aplicação no intuito de corrigir eventuais assimetrias. Nesses casos, a dose recomendada é de $5 \mathrm{U}$ no lado de maior contração muscular. No presente caso, apenas uma aplicação da BTX-A foi utilizada (10 U bilateralmente), a paciente foi reavaliada em trinta dias. Após esse período foi observado um ganho de $4 \mathrm{~mm}$ de sobreposição tecidual sobre o rebordo gengival (Figura 8). Não houve necessidade de nova aplicação da BTX-A. Após três meses de acompanhamento, a paciente manteve o mesmo nível de exposição gengival obtido com o tratamento. Convém ressaltar que este resultado tenderá a se modificar devido ao mecanismo farmacológico da BTX-A e que em um período médio de 6 meses, segundo nossos achados clínicos, a paciente necessitará nova aplicação do produto.

\section{Considerações finais}

$\mathrm{O}$ sorriso gengival, quando corretamente diagnosticado, pode ser tratado com a utilização da BTX-A.

Durante o diagnóstico e o planejamento das reabilitações orais, o CD deve considerar os padrões de beleza atuais, pois o paciente pode não estar buscando apenas dentes que reproduzam as chaves de oclusão, cor e forma propostos na literatura, mas, também, uma face harmônica e equilibrada.

A utilização da BTX-A nos pacientes diagnosticados com hipercontração da musculatura labial é uma técnica segura e reversível que pode ser indicada tanto como tratamento definitivo, quanto adjuvante a tratamentos que envolvam reabilitação orto-cirúrgica da face.

\section{Abstract}

Objective: smiling is the most beautiful facial expression and is directly connected to well-being, happiness, pleasure, and more. Physiologically, it is the result of teeth and gum exposure during the contraction of a muscle group. When smiling, many people show an excessive amount of gum, which is an esthetic problem. The aim of this study is to review the literature on etiology and treatment of the gummy smile, as well as to report through a clinical case the recommendation of botulinum toxin as a form of treatment. Case report: female patient, 22 years old, with gummy smile caused by hypermobility of the upper lip elevator muscle, gum bilateral exposure of $4 \mathrm{~mm}$. Patient was treated with botulin toxin type $A, 10 U$, bilaterally, which resulted in a harmonious and more pleasantly esthetic smile. Final considerations: gummy smile is considered an esthetic disorder by many patients and it may be treated with the use of botulinum toxin.

Keywords: Gummy smile. Botulinum toxin type A. Esthetics.

\section{Referências}

1. Oliveira M, Molina G, Molina R. Sorriso gengival, quando a toxina botulínica pode ser utilizada. Rev Odontol Araç 2011;32(2):58-61.

2. Mazzuco R, Hexsel D. Gummy smile and botulinum toxin: A new approach based on the gingival exposure area. J Am Acad Dermatol 2010;63:1042-51.

3. Silva R, Carvalho P, Joly J. Planejamento Estético em Periodontia: In: Congresso internacional de odontologia de São Paulo, 25. 2007. p. 299-341.

4. Keaney TC, Alster TS. Botulinum toxin in men: review of relevant anatomy and clinical trial data. Dermatol Surg 2013;39(10):1434-43.

5. Tae-Woo K, Hyewon K. Correction of deep overbite and gummy smile by using a mini-implant with a segmented wire in a growing Class II Division 2 patient. Am J Orthodont Dentofac Orthop 2006;130:676-85. 
6. Cheng YJ, Chin-Liang Y, Jein-Wein LE, Bowman SJ. Treatment of skeletal-origin gummy smiles with miniscrew anchorage. J Clin Orthodont 2008;42:285-96.

7. Hwang WS, Hur M, Hu KS, Song W, Koh K, Baik H, et al. Surface Anatomy of the Lip Elevator Muscles for the Treatment of Gummy Smile Using Botulinum Toxin. Angle Orthod 2009;79:70-7.

8. Graco A, Tracey S. Botox and the gummy smile. Prog Orthod. 2010;11(1)76-82.

9. Mangano A, Mangano A. Current strategies in the treatment of gummy smile using botulinum toxin type A. Plast Reconstr Surg 2012;129(6):1015.

10. Pascotto RC, Moreira M. Integração da odontologia com a medicina estética. RGO - Rev Gaúcha Odontol. 2005;53(3):171-5.

11. Seixas M, Costa-Pinto R, Araújo T. Checklist dos aspectos estéticos a serem considerados no diagnóstico e tratamento do sorriso gengival. Dental Press J Orthod 2011;16(2):131-5.

12. Suzuki L, Machado AW, Bittencourt MAV. Perceptions of gingival display aesthetics among orthodontists, maxillofacial surgeons and laypersons. Rev Odonto Ciênc 2009;24(4):36771.

13. Dutra M B, Ritter D E, Borgatto A, Derech CD. Influência da exposição gengival na estética do sorriso. Dental Press J Orthod 2011;16:5111-18.

14. Geld PV, Oosterveld P, Kuijpers-Jagtman AM. Age- related changes of the dental aesthetic zone at rest andduring spontaneous smiling and speech. Eur J Orthod 2008;30:366-73.

15. Reddy PK, Nayak DG, Uppoor A. Aesthetic Crown Lengthening: a report of 3 cases. Malaysian Dent J. 2006;1:110-3.

16. Ricketts R. M. Perspectives in the clinical application of cephalometrics: the first fifty years. Angle Orthodont. 1994;51:115-50.

17. Polo M. Botulinum toxin type A in the treatment of excessive gingival display. Am J Orthod Dentofacial Orthop 2005;127:214-18

18. Carvalho R, Shimaoka A, Andrade A. O uso da Toxina Botulínica na Odontologia. Conselho Federal de Odontologia - CFO [acesso em 11 jan. 2014]. Disponível em URL: <http://cfo.org. br/wp-content/uploads/2011/05/toxina-botulinica.pdf $>$.

19. Brasil. Resolução CFO-112/2011. Baixa normas sobre a utilização da toxina botulínica e ácido hialurônico. Setembro 2011 [acesso em: 11 jan. 2014]. Disponível em URL: <http:// cfo.org.br/servicos-e-consultas/ato-normativo/?id=1585>.

20. Mock D. Botulinum Toxin and Dentistry. In: Ensuring Continued Trust. Royal College of Dental Surgeons of Ontario, Toronto, 2009;1-4

21. Sandler J, Alsayer F, Davies J. Botox: a possible new treatment for gummy smile. Virtual Journal of Orthodontics (serial online), 2007;7(4):30-34. Feb. 20, 2007 [acesso em 2 dez. 2013]. Disponível em URL: <http://www.vjo.it/wp-content/ uploads/2010/09/botox.pdf>.

22. Sidebottom AJ, Patel AA, Amin J. Botulinum injection for the management of myofascial pain in the mastigatory muscles. A prospective outcome study. Br J Oral Maxillofac Surg. 2013;51(3):199-205

23. Emara AS, Faramawey MI, Hassaan MA, Hakam MM. Botulinum toxin injection for management of temporomandibular joint clicking. Int J Oral Maxillofac Surg 2013;42(6):759-64.

24. Jankovic J. Botulinum toxin in clinical practice. Neurol Neurosurg Psychiatry 2004;75:951-57.

25. Polo M. Botulinum toxin type A (Botox) for the neuromuscular correction of excessive gingival display on smiling (gummy smile). Am J Orthod Dentofacial Orthop. 2008;133:195-203.
26. Indra AS, Biswas PP, Vineet VT. Botox as an Adjunct to Orthognathic Surgery for a Case of Severe Vertical Maxillary Excess. J Maxillofac Oral Surg 2011;10(3):266-70.

\section{Endereço para correspondência:}

Dra. Alessandra Kuhn-Dall'Magro Rua Teixeira Soares 777/ 02 e 301 - Centro 99010-080 - Passo Fundo - RS

Email: alessandrakuhn@hotmail.com

Recebido: 16/02/2014. Aceito:05/12/2014. 